\title{
Einstein-Podolsky-Rosen Paradox and Quantum Entanglement at Subnucleonic Scales
}

\author{
Zhoudunming Tu ${ }^{1,{ }^{*}}$ Dmitri E. Kharzeev, ${ }^{2,3}$ and Thomas Ullrich ${ }^{1,4}$ \\ ${ }^{1}$ Department of Physics, Brookhaven National Laboratory, Upton, New York 11973, USA \\ ${ }^{2}$ RIKEN-BNL Research Center, Brookhaven National Laboratory, Upton, New York 11973, USA \\ ${ }^{3}$ Department of Physics and Astronomy, Stony Brook University, New York 11794, USA \\ ${ }^{4}$ Department of Physics, Yale University, New Haven, Connecticut 06511, USA
}

(Received 27 May 2019; revised manuscript received 29 October 2019; accepted 24 January 2020; published 14 February 2020)

In 1935, Einstein, Podolsky, and Rosen (EPR) formulated an apparent paradox of quantum theory [Phys. Rev. 47, 777 (1935)]. They considered two quantum systems that were initially allowed to interact and were then later separated. A measurement of a physical observable performed on one system then had to have an immediate effect on the conjugate observable in the other system-even if the systems were causally disconnected. The authors viewed this as a clear indication of the inconsistency of quantum mechanics. In the parton model of the nucleon formulated by Bjorken, Feynman, and Gribov, the partons (quarks and gluons) are viewed by an external hard probe as independent. The standard argument is that, inside the nucleon boosted to an infinite-momentum frame, the parton probed by a virtual photon with virtuality $Q$ is causally disconnected from the rest of the nucleon during the hard interaction. Yet, the parton and the rest of the nucleon have to form a color-singlet state due to color confinement and so have to be in strongly correlated quantum states-we thus encounter the EPR paradox at the subnucleonic scale. In this Letter, we propose a resolution of this paradox based on the quantum entanglement of partons. We devise an experimental test of entanglement and carry it out using data on proton-proton collisions from the Large Hadron Collider. Our results provide a strong direct indication of quantum entanglement at subnucleonic scales.

DOI: 10.1103/PhysRevLett.124.062001

Introduction.-In quantum mechanics, the entanglement of the quantum states of particles implies that a measurement performed on one of the particles affects the state of the entangled particle, even when they are located at large distances. At first glance, this implies that information would have to travel faster than the speed of light, which is forbidden by special relativity; this was referred by Einstein as "spooky action at a distance". In recent years, quantum entanglement has become the base of new technology, including quantum computers [1] and quantum cryptography [2]. At the same time, studies of entanglement in hadron physics are just beginning.

The confinement of colored quarks inside hadrons provides perhaps the most dramatic example of quantum entanglement that exists in nature. Indeed, the quarks within the hadrons are not just correlated, they simply do not exist in the physical spectrum as isolated objects. The mechanism of color confinement is one of the most challenging problems in modern physics. It is universally

Published by the American Physical Society under the terms of the Creative Commons Attribution 4.0 International license. Further distribution of this work must maintain attribution to the author(s) and the published article's title, journal citation, and DOI. Funded by SCOAP ${ }^{3}$. believed that quantum chromodynamics (QCD) should correctly describe this phenomenon, but the underlying dynamics is still mysterious. We hope that recasting this problem in the language of quantum information can shed new light on it and open new venues for experimental investigations, including the one that we describe below.

Information about short-distance QCD and the parton structure is provided by hard processes, such as deep inelastic scattering (DIS) of leptons off nucleons. Hard processes are characterized by a large-momentum transfer $Q$ and probe short transverse distances $\sim 1 / Q$ inside the nucleons and nuclei. About fifty years ago, Bjorken and Paschos [3], Feynman [4], and Gribov [5] formulated the "parton model" of the nucleon that became the base for a theoretical description of hard processes. The parton model is usually formulated in a frame in which the nucleon possesses a large momentum, and the partons are viewed by an external hard probe as independent constituents that carry different fractions $x$ of the nucleon's momentum. The assumption that the quarks and gluons confined inside the nucleon may be viewed as independent is striking. The reasoning that is commonly invoked to justify this assumption is that the parton probed at a short distance $\sim 1 / Q$ is causally disconnected from the rest of the nucleon during the hard interaction. 
Nevertheless, in spite of the spectacular success of the parton model, its assumptions raise a number of conceptual questions [6]. Indeed, the hadron in its rest frame is described by a pure quantum state $|\psi\rangle$ with density matrix $\hat{\rho}=|\psi\rangle\langle\psi|$ and zero von Neumann entropy $S=$ $-\operatorname{tr}[\hat{\rho} \ln \hat{\rho}]=0$. How does this pure state evolve to a set of "quasifree" partons in the infinite-momentum frame? If the partons were truly free and thus incoherent, they should be characterized by a nonzero entropy resulting from different positioning of partons in the configuration space. Since a Lorentz boost cannot transform a pure state into a mixed one, what is the precise meaning of quasifree? What is the rigorous definition of a parton distribution when applied to a pure quantum state?

Entanglement entropy and parton distributions.Recently, it has been suggested that this apparent paradox can be resolved by the quantum entanglement of partons [6]. Indeed, consider an electron-proton scattering with momentum transfer $Q$ depicted in Fig. 1(a). It is clear that, since the transverse distance involved in this process $\sim 1 / Q$ is much smaller than the size of the proton, DIS probes only a part of the proton's wave function; let us denote it $A$. In the proton's rest frame, where it is definitely described by a pure quantum mechanical state, DIS probes the spatial region $A$ localized within a tube of radius $\sim 1 / Q$ and length $\sim 1 /(m x)$, where $m$ is the proton's mass and $x$ is the momentum fraction of the struck quark. Inclusive DIS measurements sum over the unobserved part of the wave function localized in region $B$ complementary to $A$. Hence, we have access only to the reduced density matrix $\hat{\rho}_{A}=$ $\operatorname{tr}_{B} \hat{\rho}$, but not the entire density matrix $\hat{\rho}=|\psi\rangle\langle\psi|$. The von Neumann entropy, arising from the quantum entanglement between regions $A$ and $B$, namely, $S_{A}=-\operatorname{tr}\left[\hat{\rho}_{A} \ln \hat{\rho}_{A}\right]$

(a) ep

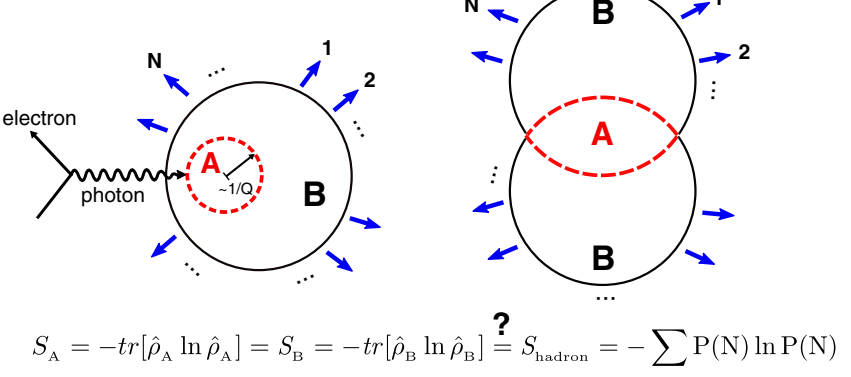

FIG. 1. Illustrations of quantum entanglement in high energy collisions. (a) Electron-proton ( $e p$ ) deeply inelastic scattering, where the virtual photon emitted by the electron probes part of the proton, denoted as region $A$, while the unobserved part of the proton is represented by region $B$. (b) Proton-proton inelastic collision, where the interaction region is $A$ and the remainder of the system is $B$. The initial von Neumann entropy from regions $A$ and $B$ is denoted as $S_{A}$ and $S_{B}$, respectively. The final-state hadron entropy $S_{\text {hadron }}$ is given by the Boltzmann entropy based on the hadron multiplicity distribution $P(N)$. associated with the DIS measurement, is found [6] to correspond to the entropy of independent partons and thus to the parton distribution. Because region $B$ is complementary to region $A$, the entanglement entropy $S_{B}$ associated with it has to be equal to $S_{A}$.

At small $x$, where gluons dominate, the relation between the entanglement entropy $S_{A}$ and the gluon distribution [7] $x G(x)$ becomes simply

$$
S_{A}=\ln [x G(x)]=S_{B}
$$

Here we do not explicitly indicate the dependence of $G$ on the momentum transfer $Q^{2}$. Equation (1) implies that at small $x$ all microstates of the system are equally probable and the von Neumann entropy is maximal.

In the present Letter, we devise an independent experimental test of measuring the entanglement entropy of partons within the nucleon using the final-state hadron multiplicity distribution $P(N)$, where $P(N)$ is the probability of producing $N$ particles in the system per event. This will allow us to test the proposed relation between the entanglement entropy and the parton distribution given by (1).

In DIS experiments, the value of the entropy arising from entanglement depends on the photon probe in terms of $x$ and $Q^{2}$. However, the entropy $S_{A}$ resulting from the entanglement of regions $A$ with $B$ and giving rise to the parton distribution should always be equal to the entropy $S_{B}$ resulting from the entanglement of region $B$ with $A$ and giving rise to the final-state entropy of the fragmenting nucleon. The latter quantity can be reconstructed from the multiplicity distribution of the produced hadrons, which we will denote as $S_{\text {hadron }}$. Based on this argument and the relation (1), we thus expect the following relation that can be directly tested in experiments:

$$
\ln [x G(x)]=S_{\text {hadron }} .
$$

This relationship can also be explored in proton-proton $(p p)$ collisions, as illustrated in Fig. 1(b). In this case, the interaction region of the two protons is region $A$, whereas the remaining system is region $B$. The signature of entanglement remains the same: the entropy reconstructed from the final-state hadrons should be equal to the entanglement entropy of the initial-state partons.

Results. - Let us begin by testing the proposed idea in the electron-proton DIS using Monte Carlo simulations. Since the probabilistic Monte Carlo event generators do not incorporate entanglement that generates entropy correlations between causally disconnected spatial domains, we do not expect relationship (2) to hold. Note that the available experimental data on hadron distributions in DIS, e.g., from hadron-electron ring accelerator (HERA) experiments, do not cover the kinematic regime of interest $\left(x<10^{-3}\right)$ where relation (2) applies. 


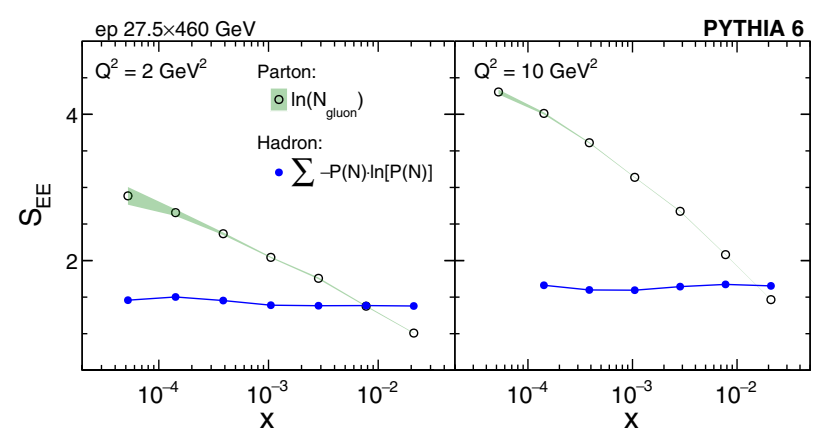

FIG. 2. Entanglement entropy in Monte Carlo generator PYTHIA6. The entanglement entropy predicted from the gluon distribution (MSTW) as a function of $x$ at momentum transfer scale $Q^{2}=2$ and $10 \mathrm{GeV}^{2}$ (open circles). The green band represents the symmetric systematic uncertainty at $90 \%$ C.L. The entropy obtained from the final-state hadron multiplicity distribution $P(N)$, from PYTHIA6 simulations at the same values of $Q^{2}$, is shown via blue filled circles. The statistical uncertainty is invisible on the scale of the plot.

First we obtain the number of gluons $N_{\text {gluon }}$ by integrating the gluon distribution $x G(x)$ over a given rapidity $y$ range at a chosen scale $Q^{2}$ (integration over $d y$ is equivalent to $d x / x$ due to change of variable). We use the leadingorder parton distribution function (PDF) set MSTW at the 90\% C.L [8], shown Supplemental Material Fig. 3 [9]. Only leading-order PDF sets are used in order to be selfconsistent with the theoretical prediction [6]. The entanglement entropy $\ln \left(N_{\text {gluon }}\right)$ predicted from the gluon distribution is shown in open black circles, with systematic uncertainties depicted as the green band in Fig. 2. The entropy of the final-state hadrons is shown as blue filled circles. It is calculated from the multiplicity distribution
$P(N)$ in a rapidity range determined by the $x$ range used to derive $N_{\text {gluon }}$. For details, see the Supplemental Material [9]. $P(N)$ is taken from ep DIS events created with the PYTHIA6 event generator [13]. We have tested several Monte Carlo event generators, such as PYTHIA6, PYTHIA8 [14], and DJANGO [15], and have found that they give similar results. An example final-state hadron multiplicity distribution is shown in Supplemental Material Fig. 4 [9].

It becomes clear from Fig. 2, that the two entropies, the von Neumann entanglement entropy associated with the gluon distribution $\ln \left(N_{\text {gluon }}\right)$, and the entropy reconstructed from the final-state hadrons $S_{\text {hadron}}$, are uncorrelated, as expected for Monte Carlo models that do not possess quantum entanglement. This correlation is absent for all MC generators that we have studied.

With a clearly drawn baseline from the Monte Carlo models, we can now look for entanglement in available experimental data. Since suitable data in $e p$ collisions do not exist, we have to turn for our study to $p p$ collisions using data from the CMS experiment [16] at the LHC. As outlined earlier, the signature of entanglement stays the same (see also Fig. 1).

By performing an analysis similar to the one presented in Fig. 2, we arrive at the results depicted in Fig. 3. Here we show the comparison of the entanglement entropy predicted from the gluon distribution (three different leadingorder PDF sets are indicated by open symbols) and the Boltzmann entropy based on the final-state hadron multiplicity $P(N)$ distribution (in filled symbols) as a function of $x$. Since $x$ and momentum transfer scale $Q^{2}$ are not directly available in $p p$ collisions (unlike in $e p$ experiments), an alternative way of comparing the entropy at similar $x$ and scales is used as detailed in the Supplemental Material [9]. The experimental data from CMS are shown in three

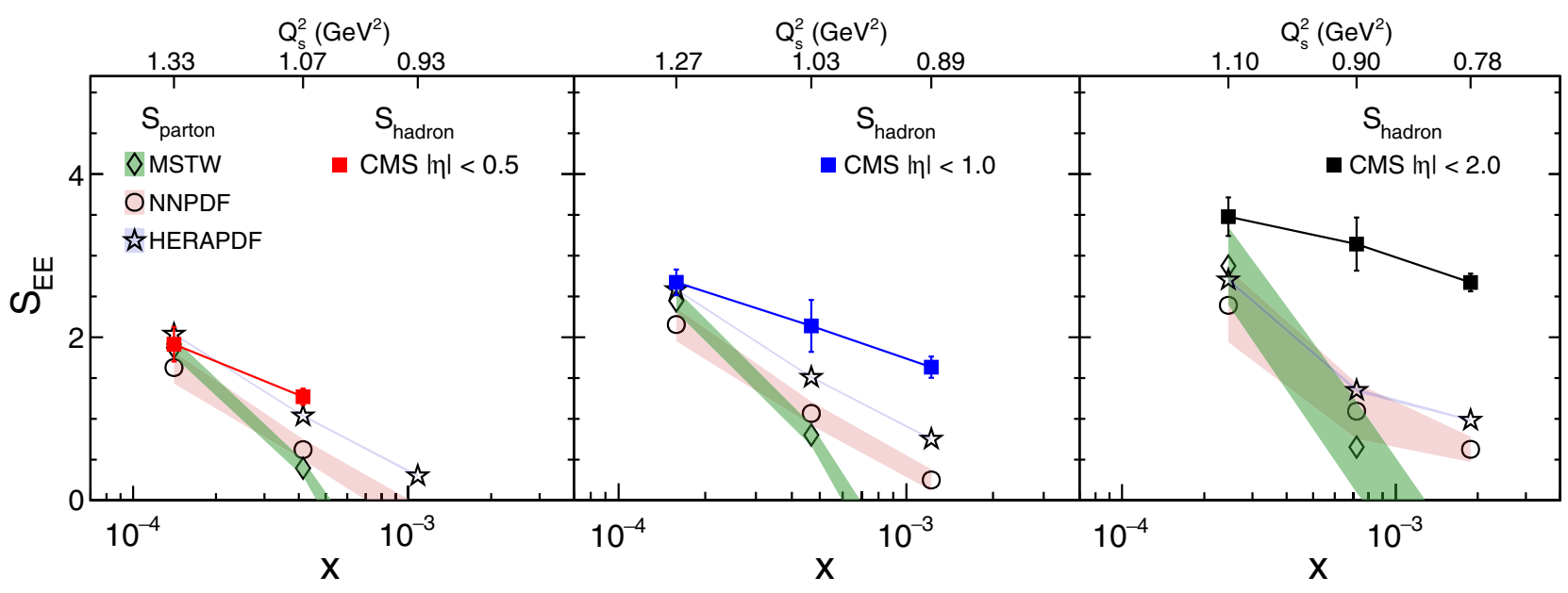

FIG. 3. Entanglement entropy in proton-proton collisions at the LHC. The Boltzmann entropy calculated based on the final-state multiplicity $P(N)$ distribution in $p p$ collisions [16] at the LHC in different pseudorapidity ranges is shown as a function of $x$ indicated by the filled squares, where the total uncertainty is denoted by the error bars. The expected entanglement entropies from the gluon distribution are presented with open markers using different PDF sets $[8,17,18]$. The bands denote the systematic uncertainty associated with the PDF. The saturation scale $Q_{s}^{2}$ [19] for gluons at each $x$ value is indicated on the top axis. 
different pseudorapidity [20] $\eta$ ranges within $\pm 0.5, \pm 1.0$, and \pm 2.0 units. Data from the pseudorapidity range within $|\eta|<1.5$ and 2.4 are depicted in Supplemental Material Fig. 1 [9]. The measurements of multiplicity distribution in $p p$ collisions performed by other LHC experiments, e.g., ATLAS [21] and ALICE [22], are consistent with CMS for the same pseudorapidity ranges where available. In this analysis, we use only CMS data since they provide a wide set of central pseudorapidity ranges not available elsewhere.

In contrast with what we have observed in $e p$ Monte Carlo simulations, we find that the experimental data from $p p$ collisions at the LHC presented in Fig. 3 show a striking agreement between the entanglement entropy predicted from the gluon distributions and the Boltzmann entropy from the final-state hadron multiplicity distributions in all $|\eta|$ ranges. Note that a constant density of hadrons per unit of rapidity usually associated with string fragmentation would imply a flat horizontal line in Figs. 2 and 3 , in conflict with the experimental data observed in $p p$ collisions. In addition, the relation (2) is expected [6] to hold for $x<10^{-3}$, and the data are in good agreement with this prediction. This observation provides a strong direct indication of quantum entanglement at subnucleonic scales. The discrepancy observed toward higher $x$ might be due to non-negligible contributions from sea quarks at low $Q^{2}$ scales. A theoretical computation of the entanglement entropy including sea quarks is not yet available and will be an important study in the future.

It is interesting to discuss the relation between the hypothesis of "local parton hadron duality" (LPHD) [23] and our results. The LPHD hypothesis states that the differential distributions of hadronic final states are proportional to the ones evaluated at the parton level, at a small virtuality scale. In particular, the number of produced hadrons is postulated to be proportional to the number of produced partons. The LPHD is well supported by the available data, but its origin remains mysterious and has been attributed [24] to the "preconfinement" property of QCD cascades $[25,26]$. The relation (2) that we have tested using the data is consistent with LPHD, provided that both the gluonic and hadron states possess the maximal entropy, i.e., are equipartitioned. It is thus possible that LPHD may have an entropic origin.

Summary.-The results reported in this Letter provide a new perspective in understanding the proton structure and shed light on the nature of color confinement. Our analysis of the experimental data from the LHC supports the resolution of an apparent paradox between the parton model and quantum mechanics based on quantum entanglement. In the future, it will be imperative to verify this result in electron-proton and electron-ion collisions at small $x$ that will require a future facility such as the electronion collider [27]. It will be also important to study the realtime evolution of quantum entanglement in high energy processes; such studies have already begun [28-31]. See the Supplemental Material for the technical details of computing entanglement entropy using partons and final-state hadrons [9].

We thank Stefan Schmitt for fruitful discussions on HERA experiments, and we thank Bertrand Ducloué and Adrian Dumitru for providing their calculations of saturation scales. The work of Z.T. is supported by LDRD-039 and the Goldhaber Distinguished Fellowship at Brookhaven National Laboratory. The work of Z. T. and T. U. is supported by the U.S. Department of Energy under Award No. DE-SC0012704. The work of D. K. was supported in part by the U.S. Department of Energy under Awards No. DE-FG-88ER40388 and No. DE-AC02-98CH10886.

zhoudunming@bnl.gov

[1] A. Steane, Rep. Prog. Phys. 61, 117 (1998).

[2] R. J. Hughes, D. M. Alde, P. Dyer, G. G. Luther, G. L. Morgan, and M. Schauer, Contemp. Phys. 36, 149 (1995).

[3] J. D. Bjorken and E. A. Paschos, Phys. Rev. 185, 1975 (1969).

[4] R. P. Feynman, Conf. Proc. C690905, 237 (1969).

[5] V. N. Gribov, Zh. Eksp. Teor. Fiz. 53, 654 (1967) [Sov. Phys. JETP 26, 414 (1968)].

[6] D. E. Kharzeev and E. M. Levin, Phys. Rev. D 95, 114008 (2017).

[7] Probability density for finding a gluon with a certain longitudinal momentum fraction $x$ at resolution scale $Q^{2}$.

[8] A. D. Martin, W. J. Stirling, R. S. Thorne, and G. Watt, Eur. Phys. J. C 63, 189 (2009).

[9] See Supplemental Material at http://link.aps.org/ supplemental/10.1103/PhysRevLett.124.062001 for computation of the entanglement entropy using initial-state partons and final-state hadrons, together with examples of parton distribution functions, saturation scales, and hadron multiplicity distributions, which includes Refs. [10-12].

[10] L. McLerran, AIP Conf. Proc. 490, 42 (1999).

[11] A. Dumitru and Y. Nara, Phys. Rev. C 85, 034907 (2012).

[12] J. L. Albacete, A. Dumitru, H. Fujii, and Y. Nara, Nucl. Phys. A897, 1 (2013).

[13] T. Sjöstrand, S. Mrenna, and P. Skands, J. High Energy Phys. 05 (2006) 026.

[14] T. Sjöstrand, S. Ask, J. R. Christiansen, R. Corke, N. Desai, P. Ilten, S. Mrenna, S. Prestel, C. O. Rasmussen, and P. Z. Skands, Comput. Phys. Commun. 191, 159 (2015).

[15] K. Charchula, G. A. Schuler and H. Spiesberger, Comput. Phys. Commun. 81, 381 (1994).

[16] V. Khachatryan et al. (CMS Collaboration), J. High Energy Phys. 01 (2011) 079.

[17] R. D. Ball et al., Nucl. Phys. B867, 244 (2013).

[18] H. Abramowicz et al. (H1 and ZEUS Collaborations), Eur. Phys. J. C 75, 580 (2015).

[19] B. Ducloué, E. Iancu, A. H. Mueller, G. Soyez, and D. N. Triantafyllopoulos, J. High Energy Phys. 04 (2019) 081.

[20] Pseudorapidity $\eta \equiv-\ln [\tan (\theta / 2)]$ and the $\theta$ is the angle with respect to the beam axis.

[21] G. Aad et al. (ATLAS Collaboration), Eur. Phys. J. C 76, 403 (2016). 
[22] S. Acharya et al. (ALICE Collaboration), Eur. Phys. J. C 77, 852 (2017).

[23] Y. I. Azimov, Y. L. Dokshitzer, V. A. Khoze, and S. I. Troyan, Z. Phys. C 27, 65 (1985).

[24] Y. L. Dokshitzer, V. A. Khoze, and S. I. Troian, J. Phys. G 17, 1585 (1991).

[25] D. Amati and G. Veneziano, Phys. Lett. 83B, 87 (1979).

[26] G. Marchesini, L. Trentadue, and G. Veneziano, Nucl. Phys. B181, 335 (1981).
[27] A. Accardi et al., Eur. Phys. J. A 52, 268 (2016).

[28] O. K. Baker and D. E. Kharzeev, Phys. Rev. D 98, 054007 (2018).

[29] J. Berges, S. Floerchinger, and R. Venugopalan, J High Energy Phys. 04 (2018) 145.

[30] A. Kovner, M. Lublinsky, and M. Serino, Phys. Lett. B 792, 4 (2019).

[31] N. Armesto, F. Dominguez, A. Kovner, M. Lublinsky, and V. Skokov, J. High Energy Phys. 05 (2019) 025. 